\title{
Time-frequency Analysis of the Autonomic Response to Head-up Tilt Testing in Brugada Syndrome
}

\author{
Mireia Calvo ${ }^{1,2,3}$, Virginie Le Rolle ${ }^{1,2}$, Daniel Romero ${ }^{1,2}$, Nathalie Béhar ${ }^{4}$, Pedro Gomis ${ }^{3,5}$, Philippe \\ Mabo $^{1,2,4}$, Alfredo Hernández ${ }^{1,2}$ \\ ${ }^{1}$ INSERM U1099, Rennes, France \\ ${ }^{2}$ LTSI, Université de Rennes 1, Rennes, France \\ ${ }^{3}$ ESAII Dept, EUETIB, CREB, Universitat Politècnica de Catalunya, Barcelona, Spain \\ ${ }^{4}$ Service de Cardiologie et Maladies Vasculaires, CHU Rennes, Rennes, France \\ ${ }^{5}$ CIBER of Bioengineering, Biomaterials and Nanomedicine, Spain
}

\begin{abstract}
Cardiac events in Brugada syndrome (BS) usually occur at rest and mainly at nighttime, commonly relating changes in autonomic modulation to arrhythmogenesis. A major challenge today is to differentiate symptomatic and asymptomatic patients in order to optimize their therapy. Since autonomic assessment can be improved by the application of standard maneuveurs, this study analyzes and compares the autonomic response to head-up tilt (HUT) testing on 20 symptomatic and 45 asymptomatic BS patients. Their standard 12-lead ECG recordings were collected during a HUT test composed of the following three phases: (1) supine rest for 10 minutes, (2) tilting with an inclination of $60^{\circ}$ for 45 minutes and (3) supine rest for 10 minutes. Spectral non-stationary heart rate variability indicators were extracted by means of a Smoothed Pseudo Wigner Ville distribution approach that adapts frequency bands to respiratory information. Symptomatic patients showed an increased parasympathetic tone and a reduced sympathovagal balance with respect to asymptomatic patients during tilting, and particularly before the $15^{\text {th }}$ minute of the test. The results provide new insights into the autonomic function in BS, with a potential impact on the use of timereduced HUT testing for risk stratification.
\end{abstract}

\section{Introduction}

Brugada syndrome (BS) is a genetic disease characterized by a distinctive ECG pattern, associated with an elevated risk for sudden cardiac death (SCD) due to malignant ventricular arrhythmias [1]. Since major cardiac events commonly occur at rest and especially during sleep, the disease is tipically related to vagal activity malfunction [2]. Moreover, data on cardiac autonomic nervous system (ANS) analyzed by positron emission tomography has evidenced that these patients display a sympathetic autonomic dysfunction [3]. Thus, changes in the autonomic modulation captured by heart rate variability (HRV) analysis may provide valuable information for risk stratification. Indeed, previous studies have already analyzed the autonomic function in BS, but they are mostly based on longterm measurements leading to contradictory results [4-7].

The autonomic response can be better characterized by stimulating the ANS through standardized maneuvers such as the head-up tilt (HUT) test. This particular stimulation provokes a redistribution of blood volume to the lower part of the body, causing a reflex sympathetic activation and a vagal inhibition that induces an increase in heart rate [8].

Although previous works have reported the role of HUT testing in BS $[5,6,9,10]$, we are not aware of any study assessing the temporal progression of these patients' autonomic function under such conditions. Thus, the aim of this work was to analyze the autonomic response to HUT testing on $65 \mathrm{BS}$ patients and compare, according to symptomatic status, the inter-patient temporal evolution of spectral HRV features resulting from time-frequency analysis.

\section{Methodology}

\subsection{Study population}

The standard 12-lead ECG recordings from 65 BS patients were collected during a HUT test divided in the following three phases: i) supine rest for 10 minutes, ii) tilting with an inclination of $60^{\circ}$ for 45 minutes and iii) rest in supine position for 10 minutes.

Patients were enrolled in 6 French university hospitals: Rennes, Saint Pierre de la Réunion, Nantes, Bordeaux, Brest and La Rochelle. After approval by the ethics committee of each center, all participants provided informed written consent to participate in the study. 
Patients' age ranged from 19 to 79 years old (46.77 $\pm 12.57)$ and $80 \%$ were males. No significant differences in age and gender between symptomatic and asymptomatic groups were noted. Twenty patients had experienced the following symptoms: syncope $(55 \%)$, cardiac arrest $(35 \%)$, dizziness (15\%), palpitations (10\%) and nocturnal convulsions $(5 \%)$. The remaining 45 patients were considered as asymptomatic.

Cardioverter defibrillator implantations (ICD) had been performed in 12 of $45(26.67 \%)$ asymptomatic patients, based on a positive EPS test, whereas all symptomatic patients were ICD carriers. Genetic testing in search of SCN5A mutations was completed in 51 patients (18 were symptomatic) and among 17 subjects in whom the $S C N 5 A$ mutation was found, 7 were symptomatic.

\subsection{Heart rate variability}

From the ECG recordings of each patient, RR-interval and R-peak amplitude series were identified by a noiserobust wavelet-based algorithm for QRS complex detection and subsequent R-peak location [11]. A cubic splines interpolation was then applied to these signals in order to obtain uniformly sampled data at a rate of $4 \mathrm{~Hz}$. To remove their very low frequency component, signals were high-pass filtered at $0.03 \mathrm{~Hz}$ with a $4^{\text {th }}$ order Butterworth filter applied in both forward and backward directions.

Given the non-stationarity of signals acquired during HUT testing, spectral HRV characteristics were analyzed by a time-frequency (TF) approach based on the Smoothed Pseudo Wigner-Ville distribution (SPWVD) transform, available from the Time-Frequency toolbox [12].

This quadratic TF method is defined as the Fourier transform of the instantaneous autocorrelation function [13], smoothed by the kernel function $\Psi(\tau, v)$, defined in Costa et al [14], that attenuates interferences while maintaining a suitable TF resolution. Being $A_{R R}(\tau, v)$ the Ambiguity Function of the RR series, $x_{R R}(t)$, the SPWVD is defined:

$$
\begin{aligned}
A_{R R}(\tau, v) & =\int_{-\infty}^{\infty} x_{R R}\left(t+\frac{\tau}{2}\right) x_{R R}^{*}\left(t-\frac{\tau}{2}\right) e^{-j 2 \pi v t} d t \\
\psi(\tau, v) & =\exp \left\{-\pi\left[\left(\frac{v}{v_{o}}\right)^{2}+\left(\frac{\tau}{\tau_{o}}\right)^{2}\right]^{2 \lambda}\right\}
\end{aligned}
$$

$C_{R R}(t, f)=\iint \psi(\tau, v) A_{R R}(\tau, v) e^{j 2 \pi(t v-\tau f)} d v d \tau$

HRV was estimated as the total power in LF and HF bands (noted as $L F b$ and $H F b$ ), extracted from the SPWVD. Assuming that sympathetic activity always lies within the standard LF band, $L F b$ was fixed between 0.04 and $0.15 \mathrm{~Hz}$ for the whole test. However, since the total power in the HF band should capture parasympathetic activity, closely related to respiration, and respiratory frequency during an ANS stimulation may not be restricted to the classic $\mathrm{HF}$ band $(0.15-0.4 \mathrm{~Hz})$, fixing $H F b$ could lead to unreliable estimates of the vagal tone. To overcome this limitation, a time-varying HF band, based on an estimation of the respiration activity captured by the ECGDerived respiration (EDR) series was implemented [15].

The estimated respiration signal was extracted from the amplitude modulation of R-wave peaks [16], band-pass filtered by a $4^{\text {th }}$ order Butterworth filter between 0.15 and $0.7 \mathrm{~Hz}$, applied in both forward and backward directions, so as to remove frequencies out of the respiratory range. The same SPWVD transform used for RR series was then applied to EDR filtered signals to obtain the instantaneous respiratory frequency.

As in [17], this respiratory frequency $\hat{f}(t)$ was estimated as that presenting the largest peak in the spectrum at each time instant. Moreover, in order to avoid spurious peak detections, for each time instant $t_{k}$, the search interval was limited to frequencies between $2 \delta \mathrm{Hz}$, centered around a reference frequency $f_{r}\left(t_{k}\right):\left[f_{r}\left(t_{k}\right)-\delta, f_{r}\left(t_{k}\right)+\delta\right]$, defined as the exponential average of previous estimates:

$$
f_{r}\left(t_{k}\right)=\beta f_{r}\left(t_{k-1}\right)+(1-\beta) \hat{f}\left(t_{k-1}\right),
$$

where $\beta$ was the forgetting factor. To reduce the risk of spurious frequency detections in the initialization of $f_{r}(t)$, the first instantaneous respiratory frequency $f_{r}\left(t_{0}\right)$ was selected within the standard HF band $(0.15-0.4 \mathrm{~Hz})$. Once $f_{r}(t)$ was estimated, the time-varying HF band was defined as $H F b(t)=\left[f_{r}(t)-0.125, f_{r}(t)+0.125\right] \mathrm{Hz}$, with $t$ covering the whole test.

The obtained time series accounting for the sympathetic and parasympathetic influences of the ANS on heart rate were normalized by the total power, defined as the sum of both spectral bands $(T P(t)=L F(t)+H F(t))$, leading to the time series $L F_{n u}(t)$ and $H F_{n u}(t)$. From this definition of normalization, it should be noted that statistical results for both $L F_{n u}(t)$ and $H F_{n u}(t)$ series are identical. $\frac{L F}{H F}(t)$ was also calculated from dividing $L F(t)$ by $H F(t)$, so as to obtain the time-varying global sympathovagal balance.

Then, these HRV time series were averaged for the whole baseline period $(B)$, in supine rest before tilting. After subtracting these reference values from each time series, they were averaged in temporal nonoverlapped windows of 1 minute, leading to $\overline{\Delta L F_{n u}^{i}}$, $\overline{\Delta H F_{n u}^{i}}$ and $\overline{\Delta L F / H F^{i}}$, which stand for the time series' intra-patient mean for the following time periods: $i \in\{T 1, T 2, \cdots, T 45, S 1, S 2, \cdots, S 10\}$. $T$ refers to the tilting phase, while $S$ accounts for the supine rest after tilting period. Fig 1 illustrates those phases composing the HUT test, as well as some of the analyzed 1-min segments. 


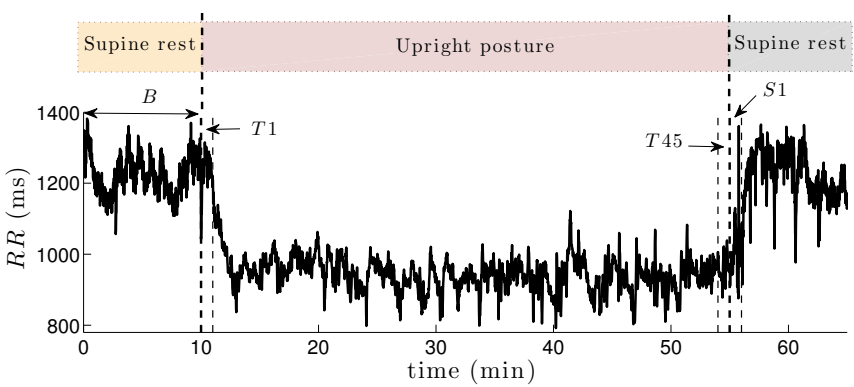

Figure 1. Representative example of RR series during HUT testing.

The extracted HRV features were finally compared between symptomatic and asymptomatic groups, by MannWhitney U non-parametric tests.

\section{Results}

Figure 2 represents the mean $\overline{\Delta H F_{n u}}$ and the mean $\overline{\Delta L F / H F}$ for both groups of patients, highlighting those 1-min segments where differences between symptomatic and asymptomatic patients were significant. It should be noted that, since the baseline value was subtracted for each patient, time series are represented in relative units (r.u.).

During tilting, $\overline{\Delta H F_{n u}}$ decreased with respect to baseline for both groups. However, symptomatic patients presented higher $\overline{\Delta H F_{n u}}$ values. Although this difference was statistically significant at different time periods, the largest segment was found before the $15^{\text {th }}$ minute in upright posture $\left(\overline{\Delta H F_{n u}^{T 11}}: p=0.030 ; \overline{\Delta H F_{n u}^{T 12}}: p=\right.$ $\left.0.031 ; \overline{\Delta H F_{n u}^{T 13}}: p=0.010 ; \overline{\Delta H F_{n u}^{T 15}}: p=0.022\right)$. After tilting, $\overline{H F_{n u}}$ was similarly restored to baseline values for both symptomatic and asymptomatic patients.

Conversely, $\overline{\Delta L F / H F}$ increased with respect to baseline in both groups during tilting, being asymptomatic patients those presenting the highest values. Statistically significant differences between groups in $\overline{\Delta L F / H F}$ were found in similar time periods than for $\overline{\Delta H F_{n u}}$, including the segment before the $15^{t h}$ minute in upright posture $\left(\overline{\Delta L F / H F^{T 13}}: p=0.034 ; \overline{\Delta L F / H F^{T 15}}: p=\right.$ 0.014). Moreover, after tilting, asymptomatic patients reached baseline $\overline{L F / H F}$ values more rapidly, leading to significant differences between groups during the third minute in post-tilt supine rest $\left(\overline{\Delta L F / H F^{S 3}}: p=0.047\right)$.

\section{Conclusion}

The time-varying autonomic response to a standardized HUT test was assessed in $65 \mathrm{BS}$ patients. According to the results, with respect to baseline, an increased parasympathetic tone $\left(\overline{\Delta H F_{n u}}\right)$ and a reduced sympathovagal balance $(\overline{\Delta L F / H F})$ were observed in symptomatic patients during tilting, in comparison to asymptomatic subjects.

Some significant differences were captured by both autonomic markers around the $35^{\text {th }}$ minute of tilting and, by the sympathovagal balance, during the third minute in supine rest after tilting, suggesting that sympathovagal balance was more rapidly restored to baseline values in asymptomatic patients. Nevertheless, since the largest and most significant segment was found before the $15^{\text {th }}$ minute in upright posture, the results suggest the potential of employing time-reduced HUT testing for risk stratification in BS. Indeed, a previous work from our team already reported the potential of 15-min HUT tests for early syncope detection [18].

Furthermore, the results confirm previous findings where symptomatic BS patients showed higher vagal $[4$, $5,7]$ and reduced sympathetic [7] tones with respect to asymptomatic patients, supporting the idea that decreased sympathetic and increased vagal responses could be related to a worse prognosis in BS.

Although comparisons are based on the symptomatic status of a relatively small population of BS patients and, thus, conclusions on the prediction of cardiac events cannot be inferred, the results indicate important trends of clinical relevance with a potential impact on the identification of BS patients at high risk.

\section{References}

[1] Brugada P, Brugada J, Roy D. Brugada syndrome 1992 2012: 20 years of scientific excitement, and more. European heart journal 2013;34(47):3610-3615.

[2] Matsuo K, Kurita T, Inagaki M, Kakishita M, Aihara N, Shimizu W, Taguchi A, Suyama K, Kamakura S, Shimomura K. The circadian pattern of the development of ventricular fibrillation in patients with brugada syndrome. European heart journal 1999;20(6):465-470.

[3] Kies P, Wichter T, Schäfers M, Paul M, Schäfers KP, Eckardt L, Stegger L, Schulze-Bahr E, Rimoldi O, Breithardt $\mathrm{G}$, et al. Abnormal myocardial presynaptic norepinephrine recycling in patients with brugada syndrome. Circulation 2004;110(19):3017-3022.

[4] Tokuyama T, Nakano Y, Awazu A, Uchimura-Makita Y, Fujiwra M, Watanabe Y, Sairaku A, Kajihara K, Motoda C, Oda $\mathrm{N}$, et al. Deterioration of the circadian variation of heart rate variability in brugada syndrome may contribute to the pathogenesis of ventricular fibrillation. Journal of cardiology 2014;64(2):133-138.

[5] Behar N, Petit B, Probst V, Sacher F, Kervio G, Mansourati J, Bru P, Hernandez A, Mabo P. Heart rate variability and repolarization characteristics in symptomatic and asymptomatic brugada syndrome. Europace 2016; euw224.

[6] Kostopoulou A, Koutelou M, Theodorakis G, Theodorakos A, Livanis E, Maounis T, Chaidaroglou A, Degiannis D, Voudris V, Kremastinos D, et al. Disorders of the autonomic nervous system in patients with brugada syndrome: a pilot 

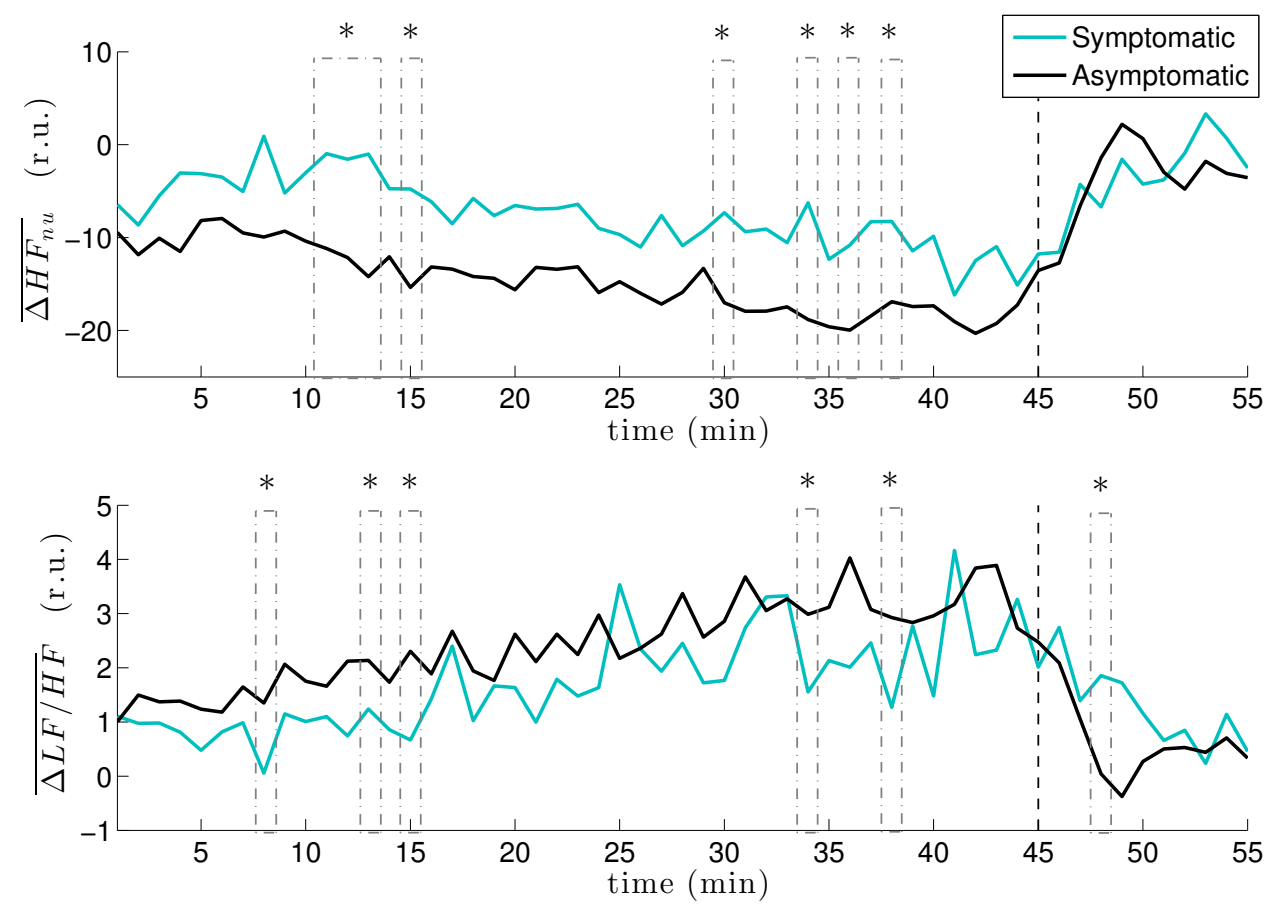

Figure 2. Mean $\overline{\Delta H F_{n u}}$ (upper panel) and mean $\overline{\Delta L F / H F}$ (lower panel) for both symptomatic (blue) and asymptomatic (black) groups, during HUT testing. Dashed vertical lines indicate the time instant where the patient changes from upright posture to supine rest. Pointed boxes delimit those 1-min segments significantly different between groups $\left({ }^{*} p<0.05\right)$.

study. Journal of cardiovascular electrophysiology 2010; 21(7):773-780.

[7] Nakazawa K, Sakurai T, Takagi A, Kishi R, Osada K, Nanke T, Miyake F, Matsumoto N, Kobayashi S. Autonomic imbalance as a property of symptomatic brugada syndrome. Circulation journal 2003;67(6):511-514.

[8] Kirchheim HR. Systemic arterial baroreceptor reflexes. Physiological Reviews 1976;56(1):100-177.

[9] Letsas KP, Efremidis M, Gavrielatos G, Filippatos GS, Sideris A, Kardaras F. Neurally mediated susceptibility in individuals with brugada-type ecg pattern. Pacing and clinical electrophysiology 2008;31(4):418-421.

[10] Calvo M, Le Rolle V, Romero D, Béhar N, Gomis P, Mabo P, Hernández A. Comparison of methods to measure baroreflex sensitivity in brugada syndrome. In Computing in Cardiology Conference (CinC), 2015. 2015; 245-248.

[11] Dumont J, Hernandez AI, Carrault G. Improving ecg beats delineation with an evolutionary optimization process. IEEE Transactions on Biomedical Engineering 2010; 57(3):607-615.

[12] Auger F, Flandrin P, Gonçalvès P, Lemoine O. Timefrequency toolbox. CNRS France, Rice University, 1996.

[13] Hlawatsch F, Boudreaux-Bartels GF. Linear and quadratic time-frequency signal representations. IEEE signal processing magazine 1992;9(2):21-67.

[14] Costa AH, Boudreau-Bartels G. Design of time-frequency representations using a multiform, tiltable exponential kernel. IEEE Transactions on Signal Processing 1995; 43(10):2283-2301.

[15] Bailón R, Laguna P, Mainardi L, Sornmo L. Analysis of heart rate variability using time-varying frequency bands based on respiratory frequency. In Engineering in Medicine and Biology Society, 2007. EMBS 2007. 29th Annual International Conference of the IEEE. 2007; 6674-6677.

[16] Moody GB, Mark RG, Bump MA, Weinstein JS, Berman $\mathrm{AD}$, Mietus JE, Goldberger AL. Clinical validation of the ecg-derived respiration (edr) technique. In Computers in Cardiology, volume 13. 1986; 507-510.

[17] Bailón R, Sornmo L, Laguna P. A robust method for ecgbased estimation of the respiratory frequency during stress testing. IEEE transactions on biomedical engineering 2006; 53(71273-1285).

[18] Khodor N, Carrault G, Matelot D, Amoud H, Khalil M, du Boullay NT, Carre F, Hernández A. Early syncope detection during head up tilt test by analyzing interactions between cardio-vascular signals. Digital Signal Processing 2016;49:86-94.

Address for correspondence:

Virginie Le Rolle

LTSI, Université de Rennes 1

Campus de Beaulieu, Bat. 22, 35042 Rennes, FRANCE virginie.lerolle@univ-rennes1.fr 